\title{
Método de dosagem adaptado para peças pré-fabricadas de concreto permeável
}

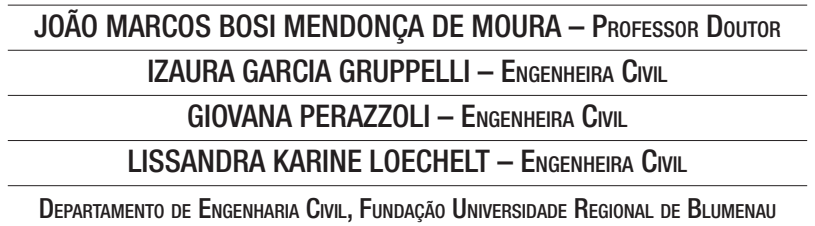

\section{RESUMO}

APRESENTA-SE UMA PROPOSTA DE ADAPTAÇÃO DA ETAPA EXPERIMENTAL PARA DETERMINAÇÃO DA RELAÇÃO ÁGUA/CIMENTO (A/C) DO CONCRETO PERMEÁVEL DO MÉTOdo dE DOSAGEM dE NGUYEN ET AL. (2014), cOM A SUBSTITUIÇÃO
DO USO DE PENEIRAS E MESA DE VIBRAÇÃO PELA TÉCNICA DO BALL-IN-HAND, COM VISTAS À DOSAGEM DO CONCRETO PERMEÁVEL PARA PRODUÇÃO DE PEÇAS PRÉ-FABRICADAS, QUE ATENDAM A ABNT NBR 16416:2015.

Palavras-chave: concreto permeável, peças pré-fabricadas, dosagem do concreto, pavimentos.

\section{INTRODUÇÃO}

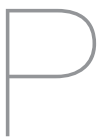

avimentos permeáveis permitem a infiltração e reserva da água pluvial, reduzindo o risco de alagamentos no ambiente urbano. $O$ concreto permeável costuma ser utilizado para revestir esses pavimentos. O material deve resistir aos esforços mecânicos e permitir a infiltração da água por meio de uma estrutura porosa, que geralmente varia entre 15 e $25 \%$ do seu volume (NGUYEN et al., 2014).

$\mathrm{Na}$ dosagem do concreto permeável deve-se levar em conta a necessidade de equilibrar o desempenho mecânico e hidráulico. Esse equilíbrio pode ser alcançado com o uso de métodos de dosagem. O método de Nguyen et al. (2014) contempla uma etapa experimental para a determinação da relação água/cimento $(\mathrm{a} / \mathrm{c})$ conhecida como teste do escorrimento do ligante (TEL) (ou Binder Drainage Test). Nele se ava- lia a consistência de pequenas misturas de concreto permeável para a escolha da relação a/c.

A técnica proporciona bons resultados para traços executados em laboratório ou in loco (NGUYEN et al., 2014; CARLS, 2018), contudo estudos preliminares indicam que a relação a/c definida não é adequada para as condições de produção de peças pré-fabricadas de concreto permeável. O maior volume de vazios reduz a coesão da mistura e dificulta desmoldagem instantânea das peças. Por isso a relevância de se buscar um método de dosagem adaptado para produção de peças pré-fabricadas de concreto permeável.

O presente estudo propõe uma ajuste ao método de dosagem de Nguyen et al. (2014) para dosagem de peças pré-fabricadas de concreto permeável. Uma modificação do TEL é realizada e validada por meio de en- saios laboratoriais. O uso das peças desenvolvidas é vantajoso porque proporciona maior padronização e controle do processo de fabricação, reduzindo incertezas durante a execução da obra.

\section{MATERIAIS E MÉTODO}

A proposta de método de dosagem adaptado para peças pré-fabricadas de concreto permeável se baseia no estudo de Nguyen et al. (2014). Propõe-se uma modificação do TEL para que a relação a/c determinada ofereça uma adequada consistência às peças de concreto permeável. Avaliou-se a massa específica no estado fresco, a resistência à compressão e o coeficiente de permeabilidade do traço dosado (Figura 1).

\section{I Materiais}

Para a dosagem do concreto permeável foi utilizado cimento, água da 
rede pública de abastecimento, agregado graúdo e agregado miúdo. 0 cimento empregado foi o de alta resistência inicial (CPV-ARI), ideal para concretos pré-fabricados que necessitam ser desformados nos primeiros dias de cura.

Na Figura 2, é apresentada a distribuição granulométrica dos agregados graúdos e miúdos. O agregado graúdo utilizado teve composição monogranular: os grãos utilizados passaram pela peneira de abertura 9,50 mm e ficaram retidos na peneira de abertura igual a 4,75 mm (Figura 2). A massa específica do agregado graúdo foi de $2583 \mathrm{~kg} \cdot \mathrm{m}^{-3}$ e sua absorção de água igual a 1,76\%. O agregado miúdo teve massa específica de $2634 \mathrm{~kg} \cdot \mathrm{m}^{-3}$, absorção de água igual a 0,50\% e módulo de finura igual a 2,16.

\subsection{Método}

O TEL consiste em avaliar o escorrimento de ligante de pequenas misturas de concreto permeável no estado fresco, com relação a/c entre 0,28 e 0,40 (em massa). Cada mistura é executada e colocada numa peneira, onde deverá ser vibrada por 15s. Se após a vibração, houver pasta de cimento no fundo da peneira, a relação a/c não é apropriada, indicando excesso de água. A relação a/c adequada será a maior possível, sem que se observe pasta no fundo da peneira após a vibração (NGUYEN et al., 2014).

Neste estudo, executou-se o TEL e a relação a/c determinada foi de 0,28. Assim, um traço dosado pelo método de Nguyen et al. (2014) com relação a/c de 0,28 , foi dosado e moldado em uma prensa hidráulica. Na Figura 3a, observa-se que a peça se desagregou após

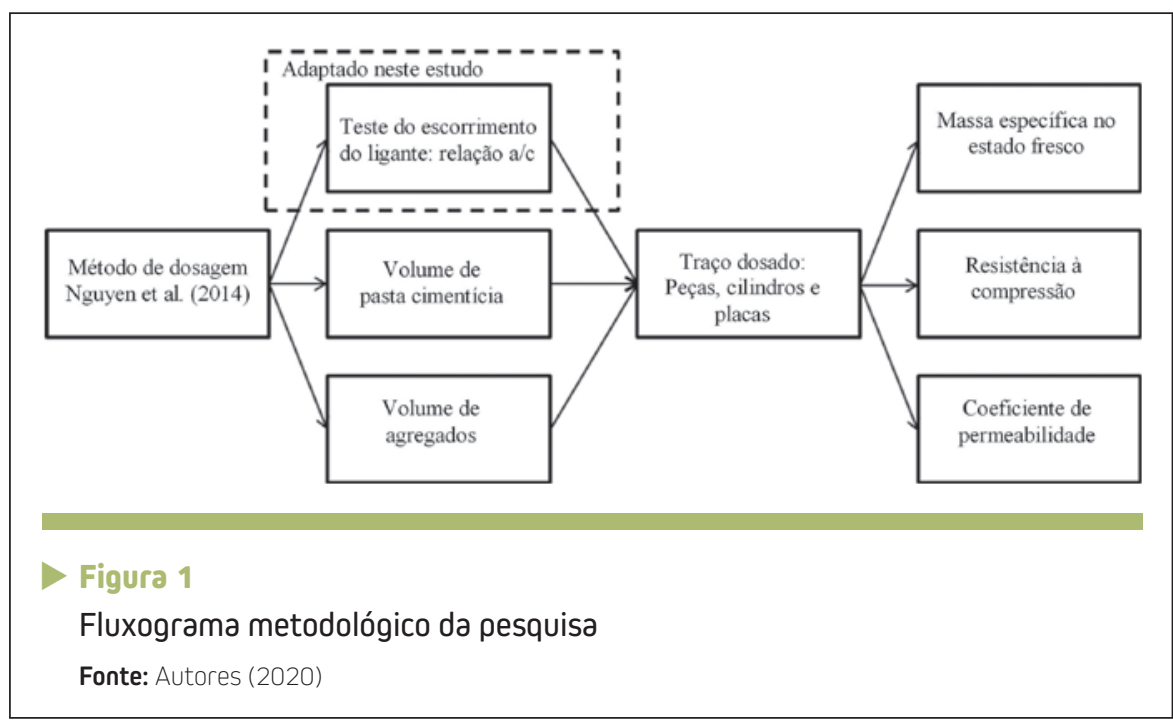

o desmolde, apontando a necessidade de se corrigir a quantidade de água.

Com o intuito de definir um procedimento objetivo para a determinação de uma relação a/c adequada para as peças, se propôs avaliar as mesmas misturas do TEL pela técnica Ball-in-hand (YAP et al., 2018). O teste permite verificar manualmente quais misturas são capazes de formar uma "bola" coesa. Assim, misturas do TEL com as relação a/c entre 0,28 e 0,32 foram moldadas pela técnica de Yap et al. (2018), em forma de uma "bola", conforme a Tabela 1. O novo critério proposto para a escolha da relação a/c de concretos permeáveis destinados a peças pré-fabricadas consiste em identificar a mistura com a menor relação a/c possível, capaz de formar uma "bola" coesa.

$\mathrm{Na}$ Tabela 1, observa-se que o traço com relação a/c de 0,28 apresentou pouca coesão, com materiais "desprendidos", o que justifica o comportamento observado na prensa (Figura 3a). Embora a relação a/c de 0,32 tenha apresentado coesão (Tabela 1), o traço teve consistência "pegajosa", gerada pela maior quantidade de água

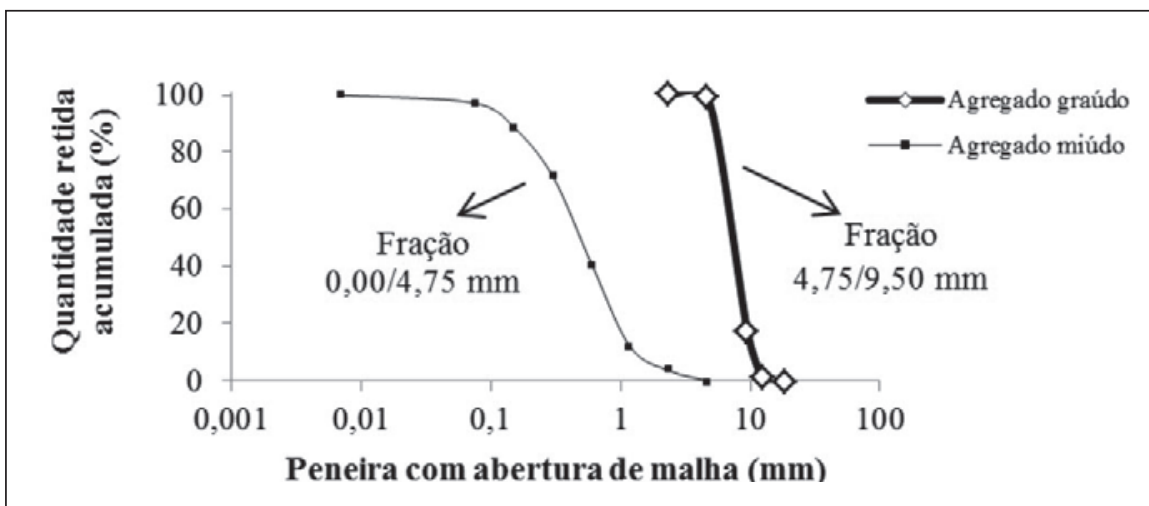

Figura 2

Distribuição granulométrica dos agregados graúdos e miúdos: frações utilizadas Fonte: Autores (2020) 


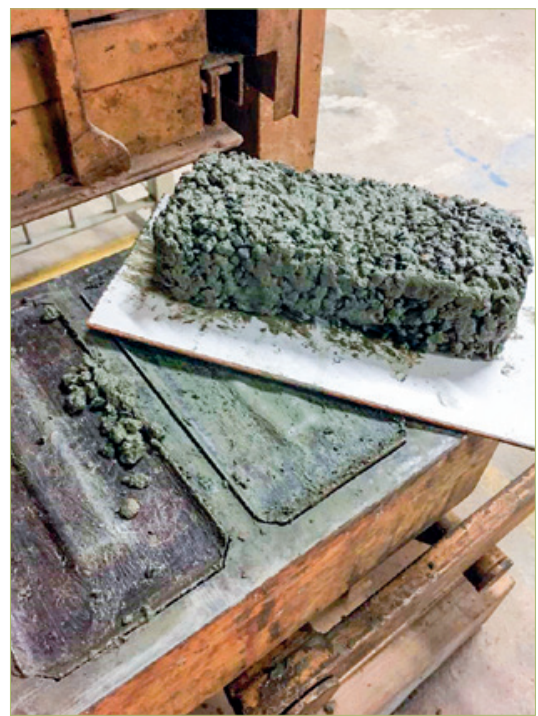

(a)

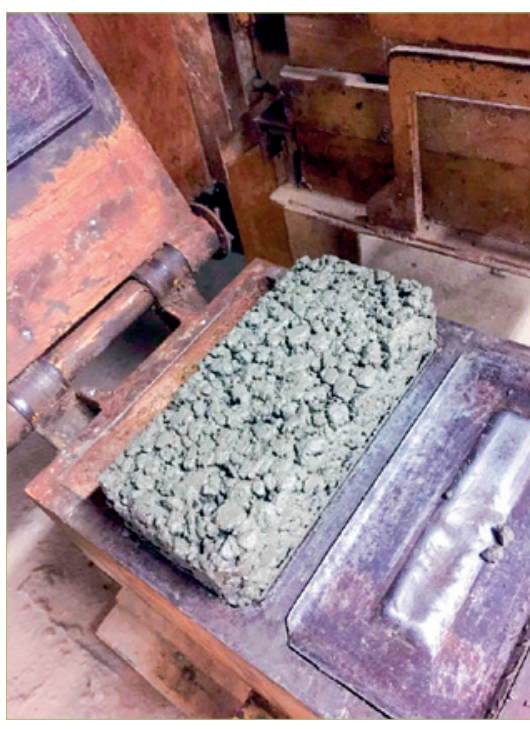

(b)

\section{Figura 3}

Execução de peça de concreto permeável: a) com relação a/c $=0,28$, obtida pelo TEL original; b) com relação a/c = 0,30, obtida por meio da modificação do TEL

Fonte: Autores (2020)

livre na mistura, dificultando também a desmoldagem no estado fresco. Esses comportamentos reforçam o critério proposto e demonstram que há uma relação a/c de equilíbrio. A menor relação a/c capaz de formar uma "bola" coesa foi igual a 0,30 (Tabela 1). O traço foi validado experimentalmente na prensa hidráulica (Figura 3b).

$O$ volume de agregado e de pasta de cimento para o concreto permeável dosado foi determinado conforme Equação 1 e 2, respectivamente (NGUYEN et al., 2014). O volume de vazios $\left(V_{v}\right)$ adotado foi igual a $20 \%$ e a relação adotada entre agregado graúdo e miúdo foi igual a quatro. A composição do concreto permeável dosado para peças pré-fabricadas é apresentada na Tabela 2.

$\mathrm{V}_{\mathrm{g}}=\frac{1-\mathrm{V}_{\mathrm{V}}}{\left(\mathrm{k}^{3}-1\right)+1+\mathrm{b}}$

Tabela 1 - TEL modificado para dosagem de peças de concreto permeável: definição da relação a/c

\begin{tabular}{|c|c|c|c|c|c|c|c|c|}
\hline$a / c$ & $\begin{array}{c}\text { C } \\
(g)\end{array}$ & $\begin{array}{c}a \\
(g)\end{array}$ & $\begin{array}{c}\text { G } \\
(g)\end{array}$ & $\begin{array}{l}\text { m } \\
(\mathrm{g})\end{array}$ & $\begin{array}{l}a_{\text {sat }} \\
(g)\end{array}$ & $\begin{array}{c}\text { Total } \\
\text { (g) }\end{array}$ & $V_{g} N_{p}$ & Ball-in-hand \\
\hline 0,28 & 133,2 & 37,3 & 400 & 100 & 7,0 & 677,5 & & \\
\hline 0,30 & 129,0 & 38,7 & 400 & 100 & 7,0 & 674,7 & 2,23 & \\
\hline 0,32 & 125,0 & 40,0 & 400 & 100 & 7,0 & 672,0 & & \\
\hline
\end{tabular}

c: cimento; a: água de amassamento; G: agregado graúdo; m: agregado miúdo; $\mathrm{a}_{\text {sat: }}$ água equivalente à absorção de água dos agregados; $\mathrm{V}_{\mathrm{g}}$ : volume de agregados; $\mathrm{V}_{\mathrm{p}}$ : Volume de pasta. Fonte: Autores (2020) 
Tabela 2 - Composição do traço dosado para peças de concreto permeável

\begin{tabular}{|c|c|c|c|c|c|c|c|}
\hline \multirow[t]{2}{*}{$\mathrm{a} / \mathrm{c}$} & \multirow{2}{*}{$V_{v}$} & Cimento & Água & $\begin{array}{c}\text { Agregado } \\
\text { graúdo }\end{array}$ & $\begin{array}{l}\text { Agregado } \\
\text { miúdo }\end{array}$ & $\begin{array}{l}\text { Água de } \\
\text { saturação }\end{array}$ & $\gamma_{\text {teórica }}$ \\
\hline & & \multicolumn{6}{|c|}{$\left(\mathrm{kg} \cdot \mathrm{m}^{-3}\right)$} \\
\hline 0,30 & 0,20 & 339 & 102 & 1176 & 295 & 22 & 1934 \\
\hline
\end{tabular}

a/c: água/cimento; $V_{v}$ : volume de vazios; $\gamma_{\text {teórica }}$ : massa específica teórica.

Fonte: Autores (2020)

$\mathrm{V}_{\mathrm{p}}=\frac{3 \cdot(\mathrm{k}-1)}{\mathrm{a} \cdot\left(\mathrm{k}^{3}-1\right)+1+\mathrm{b}} \cdot\left(\frac{\mathrm{k}+1}{2}\right)^{2} \cdot\left(1-\mathrm{V}_{\mathrm{V}}\right)$

Sendo, $V_{g}\left(m^{3}\right)$ o volume de agregado, $\mathrm{V}_{\mathrm{v}}(<1)$ o volume de vazios, $\mathrm{k}(=1,116)$ um fator que expressa a relação entre a dimensão dos agregados e a espessura de pasta de cimento, $\mathrm{b}(<1)$ a absorção de água dos agregados, a $(<1)$ a quantidade de agregados maiores que 0,075 $\mathrm{mm}$ e $\mathrm{V}_{\mathrm{p}}\left(\mathrm{m}^{3}\right)$ o volume de pasta de cimento do concreto permeável.

A seguinte ordem de mistura foi seguida, tanto para o concreto permeável testado no TEL, como para o concreto permeável executado na betoneira: agregado graúdo seco, água de saturação dos agregados, cimento, água de amassamento e agregado miúdo. O tempo de mistura foi de 3 minutos. Terminada a execução na betoneira, moldaram-se seis corpos de prova cilíndricos $(\Phi 10,20 \mathrm{~cm})$, seis peças $(22 \times 10 \times 6 \mathrm{~cm})$ e três placas (35 × $35 \times 6 \mathrm{~cm})$ de concreto permeável. Buscou-se garantir que todos os corpos de prova tivessem massa específica no estado fresco dentro do intervalo de $\pm 80 \mathrm{~kg} \cdot \mathrm{m}^{-3} \mathrm{em}$ relação à massa específica teórica - recomendação da ACl (2010).

A resistência à compressão em corpos de prova cilíndricos foi realizada de acordo com a NBR 5739:2018 e, para peças, conforme a NBR 9781:2013. As amostras submetidas aos testes mecânicos foram capeadas com pasta de cimento (relação $\mathrm{a} / \mathrm{c}=0,40)$. O coeficiente de permeabilidade foi executado nas placas segundo a NBR 16416:2015 e os procedimentos são descritos no estudo de Moura et al. (2019).

\section{RESULTADOS E DISCUSSÃO}

Os resultados dos testes laboratoriais do concreto permeável dosado são apresentados na Tabela 3. Trata-se das principais propriedades do concreto permeável (ACl, 2010). A massa específica no estado fresco dos corpos de prova variou de 1875,9 a $1996,1 \mathrm{~kg} \cdot \mathrm{m}^{-3}$ e encontra-se dentro do limite tolerável de $1934 \pm 80 \mathrm{~kg} \cdot \mathrm{m}^{-3}$ (ACl, 2010). Esse controle na massa específica é importante porque diferenças expressivas poderiam prejudicar o comparativo entre os resultados obtidos em corpos de prova com diferentes formatos.
$\mathrm{Na}$ Tabela 3, observa-se que a resistência média à compressão das peças foi de 23,1 MPa, enquanto que a resistência característica à compressão $\left(f_{c k}\right)$ foi de 22,2 MPa. Segundo a NBR 16416 (ABNT, 2015), peças de concreto permeável com $6 \mathrm{~cm}$ de espessura e $\mathrm{f}_{\mathrm{ck}} \geq 20 \mathrm{MPa}$ podem ser utilizadas como revestimento de pavimentos permeáveis destinados ao tráfego de pedestres. Portanto, as peças analisadas atendem aos requisitos da norma brasileira para essas condições.

Nos corpos de prova cilíndricos, a resistência média e característica à compressão foi de 15,5 e 10,9 MPa, respectivamente (Tabela 3). A resistência média à compressão dos cilindros foi 33\% menor que a resistência média à compressão das peças. A relação entre o $f_{c k}$ obtido com os corpos de prova cilíndricos e o $f_{c k}$ obtido com as peças foi igual a 0,49 .
Tabela 3 - Desempenho do concreto permeável dosado pelo método de dosagem modificado

\begin{tabular}{|c|c|c|c|c|}
\hline \multirow{2}{*}{$\begin{array}{l}\text { Formato do } \\
\text { corpo de prova }\end{array}$} & \multirow{2}{*}{$\begin{array}{c}\text { Massa } \\
\text { específica no } \\
\text { estado fresco } \\
\left(\mathrm{kg} \cdot \mathrm{m}^{-3}\right)\end{array}$} & \multicolumn{2}{|c|}{$\begin{array}{c}\text { Resistência à compressão } \\
\text { (MPa) }\end{array}$} & \multirow{2}{*}{$\begin{array}{l}\text { Coeficiente de } \\
\text { permeabilidade } \\
\left(\mathrm{mm} \cdot \mathrm{s}^{-1}\right)\end{array}$} \\
\hline & & $\mathrm{f}_{\mathrm{c}, \mathrm{m}}$ & $f_{c, k}$ & \\
\hline Peça & 1913,8 & 23,1 & 22,2 & - \\
\hline Cilindro & 1875,9 & 15,5 & 10,9 & - \\
\hline Placa & 1996,1 & - & - & 10,7 \\
\hline
\end{tabular}


O resultado indica uma influência importante do formato do corpo de prova na resistência à compressão medida, bem como uma relação diferente daquela que usualmente se atribuiu aos concretos convencionais (NEVILLE; BROOKS, 2013). Embora ambos os corpos de prova tenham o mesmo concreto permeável, as amostras cilíndricas possuem maior esbeltez, o que, em parte, explica a sua menor resistência à compressão. A influência da forma do corpo de prova é um aspecto importante para ser levado em conta nos estudos que avaliam dosagens preliminares de peças de concreto permeável.

Também na Tabela 3, observa-se que o coeficiente de permeabilidade medido nas placas foi de 10,7 mm.s ${ }^{-1}$, valor superior a $1 \mathrm{~mm} . \mathrm{s}^{-1}$, o mínimo exigido pela NBR 16416 (ABNT, 2015). A diferença encontrada em relação ao mínimo exigido pela norma brasileira é recomendável, uma vez que o coeficiente de permeabilidade sofre redução ao longo do tempo devido à colmatação dos poros do concreto permeável. A colmatação ocorre devido aos sedimentos transportados pela água pluvial. Kumar et al. (2016) apontam que concretos permeáveis com similar coeficiente de permeabilidade devem ser limpos a cada quatros anos. A desobstrução do revestimento pode ser realizada com equipamentos de sucção ou água sob pressão.

\section{CONCLUSÕESE RECOMENDAÇÕES}

A proposta de adaptação do método de dosagem de Nguyen et al. (2014) procurou estabelecer técnicas e critérios para a dosagem de peças de concreto permeável adequadas à NBR 16416:2015. A substituição do uso de peneiras e mesa de vibração pela técnica do Ball-in-hand foi eficaz e tornou mais simples o processo de determinação da relação a/c.

O método se mostrou adequado para as condições de estudo. O desempenho medido indica um equilíbrio entre as propriedades mecânicas e hidráulicas do concreto permeável. As peças podem ser utilizadas como revestimento de pavimento permeável em vias de tráfego de pedestres, como calçadas e parques, contribuindo para um manejo de água pluvial sustentável. Novas pesquisas podem utilizar o método proposto para verificar sua eficácia em outras condições, utilizando diferentes cimentos, agregados ou outras prensas (ex.: vibroprensa).

A infiltração da água pluvial no pavimento permeável permite criar diversos benefícios ambientais, tais como: a recarga de lençol freático, retenção de poluentes da água pluvial e recuperação das condições hidrológicas naturais. Por esta razão, a manutenção periódica das peças deve ser realizada para que o coeficiente de permeabilidade do revestimento seja preservado no local de aplicação.

Assim, o estudo abre caminho para que a produção de peças de concreto permeável seja realizada com maior eficiência e qualidade, contribuindo para a promoção de técnicas do manejo de água pluvial sustentável.

\section{AGRADECIMENTOS}

O presente trabalho foi realizado com apoio da Coordenação de Aperfeiçoamento de Pessoal de Nível Superior - Brasil (CAPES) - Código de Financiamento 001.

\section{> REFERÊNCIAS BIBLIOGRÁFICAS}

[1] ABNT - Associação Brasileira de Normas Técnicas.NBR 16416 - Pavimentos permeáveis de concreto - Requisitos e procedimentos. Rio de Janeiro: 2015.

[2] $\mathrm{ACl}$ - American Concrete Institute. Specification for Pervious Concrete Pavement. 522R-10.1-13. ISBN: 9780870318221. 2010. 38pp.

[3] CARLS, Bruna. Coeficiente de permeabilidade, resistência mecânica e durabilidade de concreto permeável dosado por três diferentes métodos. 2018.109 p., il. Dissertação (Mestrado em Engenharia Ambiental) - Programa de Pós-Graduação em Engenharia Ambiental, Centro de Ciências Tecnológicas, Universidade Regional de Blumenau, Blumenau, 2018. Disponível em: http://www.bc.furb.br/docs/DS/2018/365221_1_1.pdf. Acesso em: 20 maio 2020.

[4] KUMAR, Kuldip et al. In-situ infiltration performance of different permeable pavements in an employee used parking lot-A four-year study. Journal of Environmental Management, v. 167, p. 8-14, 2016, https://doi.org/10.1016/j.jenvman.2015.11.019.

[5] MOURA, J. M. B. M.; PERAZZOLI, G.; HAMANN, C.; OLIVEIRA, J. P. Análise do coeficiente de permeabilidade de revestimentos cimentícios e suas implicações para gestão urbana. In: XXIII SIMPÓSIO BRASILEIRO DE RECURSOS HÍDRICOS, 2019, Paraná. Anais... Foz do Iguaçu: ABRHidro, 2019. Disponível em:< http://anais. abrh.org.br/works/6535>.

[6] NEVILLE, Adam M; BROOKS, J. J. Tecnologia do concreto.2. Ed. Porto Alegre: Bookman, 2013. 448 p, il.

[7] NGUYEN, Dang Hanhet al. A modified method for the design of pervious concrete mix. Construction and Building Materials, v. 73, p. 271-282, 2014, https://doi. org/10.1016/j.conbuildmat.2014.09.088.

[8] YAP, SoonPoh et al. Characterization of pervious concrete with blended natural aggregate and recycled concrete aggregates. Journal of Cleaner Production, v. 181 p. 155-165, 2018, https://doi.org/10.1016/j.jclepro.2018.01.205. 